\title{
A comparison study of two breathing exercise techniques in tetraplegics
}

\author{
Stanley John Winser ${ }^{1}$, Jacob George ${ }^{2}$, Priya Stanley ${ }^{1}$, George Tharion ${ }^{2}$ \\ ${ }^{1}$ Masterskill University of Health Science (MUCH), Cheras, Malaysia; stanjwpt@gmail.com, stanjw_pt@yahoo.com, \\ stanley@masterskill.edu.my \\ ${ }^{2}$ Christian Medical College, Vellore, India.
}

Received 15 July 2009; revised 23 July 2009; accepted 25 July 2009.

\section{ABSTRACT}

Objective: To compare the effectiveness of abdominal weights and incentive spirometry for improving the strength of diaphragm in tetraplegics.

Setting: Department of Physical Medicine and Rehabilitation, Christian Medical College, VelIore, Tamil Nadu, India.

Study Design: Two group comparison study

Methods: Seventeen patients who fulfilled the inclusion criteria were assigned into an $A B W$ or INS treatment groups using judgment sampling after obtaining an informed consent. Evaluation of the chest, respiratory status, vital signs and strength of diaphragm were done during initial assessment. ABW group underwent diaphragmatic strengthening using Abdominal weights (ABW) and INT group with Incentive Spirometer (INS) for 15 minutes daily, 6 days a week, for a period of 6 weeks. The pre and post training values of peak amplitude in electro myogram (EMG) of the diaphragm, intercostals and sternocleidomastoid muscles were measured.

Statistical analysis: The analysis was done using SPSS 11. The pre and post-training values of peak EMG amplitudes of the diaphragm, intercostals and sternocleidomastoid were compared within the groups using Wilicoxon's sign test and between the two groups using MannWhitney's test.

Results: The peak EMG of diaphragm of ABW group raised from 1.1289 to 1.3036 milli-volts with a significance of $p<0.001$, whereas it fell from 1.7001 to 1.0441 milli-volts among INS group subjects with a significance of $p<0.001$. Comparison between the 2 groups showed statistically significant improvement in diaphragmatic strength among the ABW group.

Conclusion: The results of this study suggests that, in the pulmonary rehabilitation of motor complete tetraplegic subjects abdominal weighted training of the diaphragm has better results in improving the strength of the muscle. Sponsorship: Fluid research grant of Christian Medical College, Vellore.

Keywords: Spinal Cord Injury; Tetraplegia; Diaphragmatic Exercise; Surface EMG; Abdominal Weights; Incentive Spirometer

\section{INTRODUCTION}

Spinal cord injury (SCI) at cervical level results in tetraplegia with or without paralysis of diaphragm. Among complete tetraplegics with intercostals and diaphragmatic paralysis there is severe respiratory insufficiency especially in the acute stage of illness [1]. Respiratory insufficiency leads to high mortality rate in these patients [2]. Following cervical cord injury there is paralysis of respiratory muscles [3] which leads to accumulation of secretions. Failure of cough mechanism causes recurrent infections which further compromises lung functions and add to morbidity and mortality.

Studies on pulmonary function tests of individuals with complete tetraplegia have shown that there is a major loss of expiratory reserve volume due to paralysis of expiratory muscles causing reduction of maximal expiratory pressure. The work of breathing is increased and the diaphragm is prone to fatigue particularly in patients with high cervical cord lesion [4]. Physiotherapy management strategies aspire at improving efficiency of diaphragm and training the available accessory muscles to compensate for paralyzed respiratory muscles. Progressive resisted exercises have been used in strengthening respiratory muscles for high tetraplegic patients [5]. There are various methods describe in literature to improve the efficiency of diaphragm [6], which includes Resisted Inspiratory Muscle Training (RIMT) [7,8,9], Weighted diaphragmatic exercise [10], Abdominal binders [11], Trendlenbergs position and Incentive spirometry. 
There is paucity of literature which compares the effectiveness of abdominal weighted training and incentive spirometry in improving the strength of diaphragm. This study designed at evaluating the effectiveness of two pulmonary rehabilitation programmes (weighted diaphragmatic exercise against incentive spirometry) which are commonly practiced for training the diaphragm among patients with spinal cord injury.

\section{MATERIALS AND METHODS}

This study was approved by the Institutional review board and ethics Committee of Christian Medical College. The background of the study was explained to the subjects in his ther language and the patients who were willing to participate were inducted into the study following an informed written consent. The sample size was calculated to be 6 in each group with ABW group as subjects trained with abdominal weights and INS group as subjects trained with incentive spirometry. The subjects with neurological level between C5 and C8 ASIA [12] grade A \& B, 1 month post injury, muscle strength of diaphragm more than fair plus, medically stable patients and without any current lung infections or comorbidity were included in the study. Subjects with associated Traumatic Brain Injury (TBI), past history of Chronic Obstructive Pulmonary Disease, Tuberculosis and chronic smokers were excluded.

\subsection{Sample Size \& Method}

The sample size was calculated with $5 \%$ alpha level and $80 \%$ power, with having a SD of 0.73 and 0.32 ; the sample size was found to be 6 in each group. We had adopted judgment sampling method. The subjects who underwent weighted diaphragmatic training had to lie supine. Since some subjects had pressure ulcer over the sacral region, supine lying was contraindicated, which made us to assign them into the INS group.

\subsection{Evaluation of Strength [13]}

The strength of diaphragm was graded using the grades as follows. "Poor power": is being graded if the subject is not able to expand his her epigastric region fully on deep inspiration. "Fair power": if the subject is able to expand his $\backslash$ her epigastric region fully on deep inspiration. "Good power": the therapist's hands are placed over the epigastric region with fingers spread, and the subject is asked to inhale, while maximum manual resistance is applied. If the subject is able to complete a full epigastric raise against resistance then helshe can be graded as Good. The subjects who are able to take resistance but not able to hold can be graded as "Fair plus". Subjects with diaphragmatic power of fair plus and above were considered for progressive resisted exercises. Prior to the commencement of training, the EMG activity of the diaphragm, intercostals and sternocleidomastoid muscles were evaluated.

\subsection{Outcome Measure (EMG Analysis)}

The EMG activity was measured using 3 pairs of Silver chloride bipolar surface electrode. The active electrodes were placed over T7 \& T8 intercostal space, T4 \& T5 intercostal space and mid portion of sternocleidomastoid to get the electrical activity of diaphragm, intercostals and sternocleidomastoid muscles respectively [14], readings of all three groups of muscles were taken simultaneously (see Figure 1). Electrodes were secured to the skin using adhesive plaster after skin preparation. While taking the readings the subjects were instructed to take 3 consecutive deep inspirations followed by expiration, reading were recorded for 10 seconds and the peak amplititudes in the EMG recordings noted. The investigator who performed this test was blinded to either group. Three trials were done and the best response of peak amplitude was noted.

\subsection{Intervention}

The subjects were allotted into both groups using the above mentioned sampling method. Subjects of both the groups concurrently underwent other rehabilitative programs such as passive range of motion exercise, activities of daily living, standing using tilt or standing table, strengthening or re-education for the available muscles. In the ABW group, diaphragm was strengthened using abdominal weights for 15 minutes per day for six days weekly for a period of 6 weeks. The subjects of INS group underwent training using incentive spirometer for the same period as ABW group.

\subsection{Evaluation of Weight [15]}

To train the ABW group, the appropriate weights to

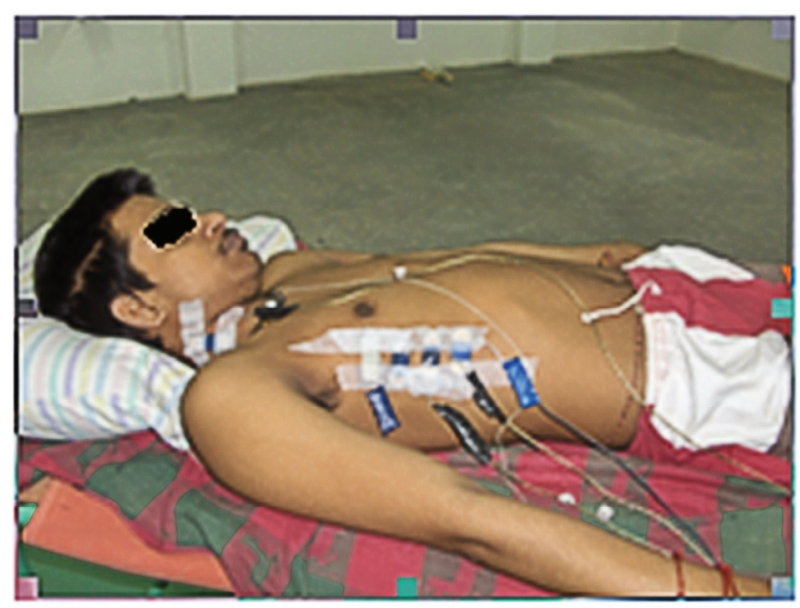

Figure 1. Showing placement of surface electrodes for the assessment of EMG activity of Intercostals, Diaphragm and Sternocliedomastoid muscles. 
strengthen the diaphragm were evaluated using the following method. The subject was positioned in supine lying, then a minimal weight was placed over the epigastric region (weights starts with half a kilogram) and then the subject was allowed to breath (the weight should come up fully with each inspiration). If the subject showed any signs of fatigue or started using his her accessory muscles, the weights were taken off immediately. Adequate rest was given, then the procedure was repeated using a lesser weight, and if the patient is able to take up the weight comfortably for 15 minutes, a short break was given and the procedure was repeated by increasing the weight by half a kilogram, thus the appropriate weight for training the diaphragm was determined by trial and error method. Using the evaluated weight the diaphragm was strengthened during the study period.

\subsection{Placement of Weight}

The evaluated weight was placed over the epigastric region with an isosceles triangular board. The board is placed in such a way that one of the corners touches the xiphisternum and the other two corners touching the anterior borders of the ribcage (see Figure 2). During the training period of the ABW group, subjects were instructed to perform normal breathing with the weights on for 15 minutes and progression in weights was done for every 1 week.

\subsection{Incentive Spirometry Training}

The INS group subjects were trained using an Incentive spirometer, it is a flow oriented breathing exerciser. The devise is provided with 3 balls and they provide the patients an indirect indicator of the inspired volume. Three color-coded balls ( 3 shades of green) in each chamber provide a visual incentive for the patient. Air flows into single channel, when it passes through the chamber, it raises each of the three balls depending on the flow inhaled per second. Flow rates of the spirometer includes $600 \mathrm{~mL} / \mathrm{sec}, 900 \mathrm{~mL} / \mathrm{sec}$ and $1200 \mathrm{~mL} / \mathrm{sec}$. By using different colors of ball it becomes easy to identify the flow rate. Subjects of this group were instructed to keep the balls of the spirometer suspended for maximum time possible, this was repeated for 15 minutes daily for a period of 6 weeks. Progression was made by increasing the suspending time of the spirometer ball.

At the end of 6 weeks training, the EMG activities of diaphragm, intercostals and the sternocleidomastoid muscles were re-examined and compared for results.

\section{STATISTICAL ANALYSIS}

The analysis was done using SPSS 11. Baseline values were tabulated as shown in Table 1.The pre and post-training values of peak EMG amplitudes of the dia-

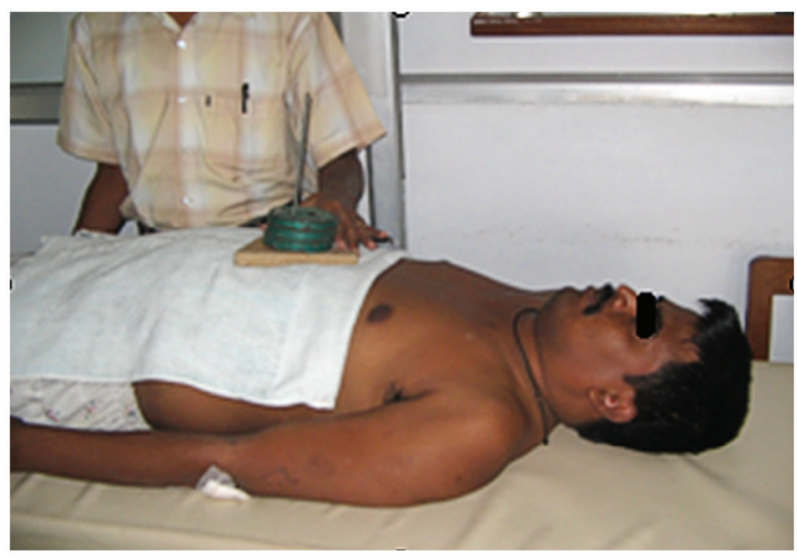

Figure 2. Diaphragmatic strengthening using abdominal weights.

Table 1. Baseline characteristics of analysed data.

\begin{tabular}{|c|c|c|c|}
\hline \multicolumn{2}{|c|}{ Characteristics } & Group (A) & Group (B) \\
\hline \multicolumn{2}{|c|}{ Mean age } & $36+/-12.32$ & $27.16+/-8.91$ \\
\hline \multirow{2}{*}{ Sex } & Male & 7 & 6 \\
\hline & Female & - & - \\
\hline \multirow{2}{*}{$\begin{array}{l}\text { Cause of } \\
\text { lesion }\end{array}$} & Traumatic & 6 & 5 \\
\hline & Non traumatic & 1 & 1 \\
\hline \multicolumn{2}{|c|}{ Time since injury (days) } & $68+/-32.72$ & $74.32+/-38.71$ \\
\hline \multirow{2}{*}{$\begin{array}{l}\text { ASIA clas- } \\
\text { sification }\end{array}$} & A & 5 & 3 \\
\hline & B & 2 & 3 \\
\hline \multicolumn{2}{|c|}{ Presence of sacral ulcers } & - & 5 \\
\hline
\end{tabular}

phragm, intercostals and sternocleidomastoid were compared within the groups using Wilicoxon's sign test and between the two groups using Mann-Whitney's test.

\section{RESULTS}

Seventeen subjects were recruited into the study with 9 patients in group A and 8 in group B. Thirteen subjects had completed the study and were available for the post training assessment and analysis. Two subjects from each group dropped out from the study. Among 4 subjects dropped out, two were not willing to undergo post training assessment, one subject got discharged against medical advice and another subject was shifted to critical care unit. The mean age of subjects was 35 years (range 19 to 53 years).

Among 17 subjects, 13 had traumatic SCI and remaining 4 patients had infective pathology of the cord. Mean duration since injury was 71.61 days (range 32 to 142). There was a significant increase in the diaphragmatic activity of subjects in the ABW group, shown in Table 2, whereas there was a significant reduction in the EMG activity among the INS group, shown in Table 3. Comparison between the 2 groups using Mann-Whit 
Table 2. Change in EMG ( milli volts) within ABW group.

\begin{tabular}{ccccccc}
\hline \multirow{2}{*}{$\begin{array}{c}\text { Group } \\
\text { A }\end{array}$} & \multicolumn{2}{c}{ Pre- test } & \multicolumn{2}{c}{ Post - test } & P & Z \\
& Mean & SD & Mean & SD & & \\
\hline T4 & 0.8478 & 0.8463 & 1.2915 & 1.5638 & $<0.001$ & -23.490 \\
T5 & 0.7253 & 0.7974 & 1.2366 & 1.4240 & $<0.001$ & -31.986 \\
T7 & 1.1289 & 0.7862 & 1.3036 & 1.2129 & $<0.001$ & -6.273 \\
T8 & 0.8188 & 0.6440 & 0.8977 & 0.9190 & $<0.001$ & -0.475 \\
SCM & 1.5027 & 1.8742 & 1.2527 & 1.5241 & $<0.001$ & -8.424 \\
\hline
\end{tabular}

Table 3. Change in EMG ( milli volts) within INS group.

\begin{tabular}{|c|c|c|c|c|c|c|}
\hline \multirow{2}{*}{$\begin{array}{c}\text { Group } \\
\text { B }\end{array}$} & \multicolumn{2}{|c|}{ Pre- test } & \multicolumn{2}{|c|}{ Post - test } & & \\
\hline & Mean & SD & Mean & SD & & \\
\hline $\mathrm{T} 4$ & 0.9554 & 0.8923 & 0.7368 & 0.7668 & $<0.001$ & -17.659 \\
\hline T5 & $1.1^{\prime}$ & 1. & 0 & 3 & $<0$. & -14 \\
\hline $\mathrm{T} 7$ & 1.7001 & 1.4144 & 0.9631 & 0.8993 & $<0.001$ & -31.584 \\
\hline $\mathrm{T} 8$ & 1.8194 & 1.8367 & 0.9823 & 0.9823 & $<0.001$ & -25.335 \\
\hline SCM & 2.3985 & 2.4365 & 2.0446 & 1.8431 & $<0.001$ & -9.838 \\
\hline
\end{tabular}

Table 4. Comparing change in EMG (milli volts) between the 2 groups.

\begin{tabular}{|c|c|c|c|c|c|c|c|c|}
\hline \multirow{2}{*}{ Muscle } & \multicolumn{2}{|c|}{ Group (A) } & \multirow{2}{*}{$P$} & \multicolumn{2}{|c|}{ Group (B) } & \multirow[t]{2}{*}{$P$} & \multicolumn{2}{|c|}{$\mathbf{Z}$} \\
\hline & Pre & Post & & Pre & Post & & Pre & post \\
\hline $\mathrm{T} 4$ & 0.8472 & 1.2915 & 0.00 & 0.9554 & 0.7368 & $<0.001$ & -14.019 & -19.676 \\
\hline T5 & 0.7253 & 1.2366 & 0.00 & 1.1756 & 0.9631 & $<0.001$ & -40.670 & -0.105 \\
\hline $\mathrm{T} 7$ & 1.1289 & 1.3036 & 0.00 & 1.7001 & 1.0441 & $<0.001$ & -23.396 & -8.030 \\
\hline $\mathrm{T} 8$ & 0.8188 & 0.8977 & 0.00 & 1.8194 & 0.9823 & $<0.001$ & -32.875 & -13.278 \\
\hline $\mathrm{SCM}$ & 1.5027 & 1.2524 & 0.00 & 2.3985 & 2.0446 & $<0.001$ & -20.671 & -27.735 \\
\hline
\end{tabular}

ney's test showed a statistical significance $(p<0.001)$ in the improvement of diaphragmatic activity in $\mathrm{ABW}$ group, shown in Table 4. The mean EMG of diaphragm of ABW group raised from 1.1289 to 1.3036 milli-volts with a significance of $\mathrm{p}<0.001$, whereas it fell from 1.7001 to 1.0441 milli-volts among INS group subjects with a significance of $\mathrm{p}<0.001$. When comparing the EMG activities of both the groups a statistically significant improvement in diaphragmatic strength was observed among the ABW group.

\section{DISCUSSION}

The objective of the study was to determine which of the two techniques, (abdominal weights or incentive spirometry) improves the strength of diaphragm among complete tetraplegic patients. Results of this study have shown that patients trained with weighted diaphragmatic exercise had better improvement in the post training assessment of EMG values. Pulmonary rehabilitation exercise protocols prescribed for the spinal cord injured patients with reduced pulmonary function is very effective to provide a positive outcome. Pulmonary exercises are simple and effective without any need to procure sophisticated instruments. Studies done on the progressive resistive exercises for the respiratory muscles have proved that this method of diaphragm training may be useful in weaning high level quadriplegic patients from the ventilator.

Studies on pulmonary function tests of individuals with complete tetraplegia showed that there is a major loss of expiratory reserve volume because of paralysis of expiratory muscles, as a result of which their maximal expiratory pressure was reduced. The vital capacity approximates their inspiratory capacity. The work of breathing is increased and the diaphragm is prone to fatigue particularly in patients with high cervical cord lesion [16].

Carolyn Kisner and Lynn Allen Colby in their discussion on various techniques used to strengthen diaphragm suggested; incentive spirometry is a form of low level resistance training that emphasizes sustained maximal inspiration. The training sessions of the above mentioned studies were typically limited to 15 to 30 minutes each, with two to three sessions a day, 5 to 7 days a week for a total period of 6 to 8 weeks.

Previous studies have compared the effectiveness of resistive inspiratory muscle training (RIMT) and abdominal weights in improving the strength of diaphragm among tetraplegic patients and have proved that both the techniques are equally effective [17]. Hwa Lin and Chy Ching Chuang observed that Abdominal weighted maximal ventilatory (AWMV) breathing evoked greater EMG activity, inspiratory flow and inspiratory volume than did (Inspiratory resistance maximal ventilatory (IRMV) breathing. The increase of diaphragmatic EMG was not statistically significant in AWMV breathing. Weighted diaphragmatic strengthening is a less commonly employed strengthening program. Studies had shown that the use of weighted diaphragmatic exercise was of great therapeutic use, and have compared the effects of abdominal weights against resistive inspiration as mentioned before. On the other hand in recent days incentive spirometry is the most commonly administered 
treatment regimen in improving the strength and efficiency of diaphragm among tetraplegics. In our study we have observed, the most efficient and cost effective modality in the pulmonary management of tetraplegic patients is abdominal weighted exercise.

We hypothesized a reduction in the EMG activity of the sternocleidomastoid muscle following training due to an improvement in the efficiency of diaphragm which is the primary respiratory muscle. We found an increase in the activity of sternocleidomastoid muscle among ABW group subjects along with the increase in activity of the other groups of muscles (intercostals and diaphragm). There was a reduction in activity of sternocleidomastoid muscle among the INS group subjects. However we could not explain the reason for this phenomenon. The results of this study are not concurrent with the previous literature; instead has proved that resistive diaphragmatic breathing exercise has better therapeutic effects in improving the strength of diaphragm among complete tetraplegic patients.

There were few limitations in our study which includes the sampling technique and spasticity of the subjects. There are possibilities that disturbances from the abnormally activated trunk muscles could have reduced the accuracy of EMG activity of the diaphragm and other respiratory muscles. The sampling method had to be judgement sampling rather than single blinded simple random sampling which could have been ideal for this study.

\section{CONCLUSIONS}

The peak EMG amplitude showed a significant rise amongst subjects who were trained using abdominal weights. This is an apparent indication of improved performance of the target muscle with regards to strength. Cost effectiveness and ease of administration of this technique ensures its frequent usage by clinical practitioners. Thus we conclude stating Abdominal weights can be used as an effective adjunct to pulmonary rehabilitation in improving the strength of diaphragm, thereby reducing the risks associated with pulmonary complications.

\section{ACKNOWLEDGEMENT}

We gratefully acknowledge Mr. Ganesh, Movement Analyst who assisted us in collecting EMG recordings for all our patients and $\mathrm{Mr}$. Vijayakumar for his guidance. We also acknowledge the Fluid research committee for funding our project.

\section{REFERENCES}

[1] DeVivo, M.J., Black, K.J., and Stover, S.L. (1993) Causes during first twelve years after spinal cord injury. Arch Phys Med Rehabil, 74, 248-54.

[2] Liaw, M.-Y., Lin, M.-C., and Cheng, P.-T. (2000) Resistive inspiratory muscle training: its effectiveness in patients with acute complete cervical cord injury. Arch. Phys. Med Rehabil, 81, 747-751.

[3] Garshiek, K.A. and Gross, E.R. (2003) Spirometry testing standards in spinal cord injury. Chest, 123, 725-730.

[4] Ledsome, J.R. and Sharp, J.M. (1981) Pulmonary functions in acute cervical cord injury. Am Rev Respir Dis, 124, 41-44.

[5] Lerman, R.M. and Weiss, M.S. (1987) Progressive resistive exercise in weaning high quadriplegic from the ventilator. Paraplegia, 25(2), 130-5.

[6] Kisner, C. and Colby, L.A.. In: Therapeutic Exercises. Foundation and techniques, 3rd edn, 665-672.

[7] Gross, D., Ladd, H.W., Riley, E.J. (1980) The effect of training on strength and endurance of the Diaphragm in quadriplegics. The American journal of Medicine, 68 (1), 27-35.

[8] Wang, T.G., Wang, Y.H. (2002) Resistive inspiratory muscle training in sleep disordered breathing of traumatic tetraplegia. Arch Phy Med Rehabil, 83(4), 491496.

[9] Rutchik, A., Weissman, A.R. (1998) Resisitive inspiratory muscle training in subjects with chronic spinal cord injury. Arch Phy Med Rehabil, 79 (3), 293-297.

[10] Lane, C.S. (1982) Inspiratory muscle weight training and its effect on the vital capacity of patients with quadriplegia. Boston (MA) Northern University.

[11] Bodin, P., Fagevik Olsen, M., Bake, B. (2005) Effects of abdominal binding on breathing pattern during breathing exercises in persons with tetraplegia. Spinal Cord, 43, 117-122.

[12] American spinal injury assosiation; standard for neurological and functional classification of SCI. (1992) Revised 1992 Chicago ASIA.

[13] Wetzel, J. L., Lunsford, B. R. In: Scot Irwin, Teclin (ed): Cardio Pulmonary Physical therapy, 3rd edn, 584-586.

[14] Lin, H. and C.-C. Chung. (1999) Abdominal weight and Inspiratory resistance: Their immediate effects on inspiratory muscle functions during maximal voluntary breathing in chronic tertraplegic patients. Arch. Phys. Med. Rehabil, 80(7), 741-745.

[15] Wetzel, J.L. and Lunsford, B.R. In: Scot Irwin, Teclin (ed): Cardio Pulmonary Physical therapy, 3rd edn, 588589.

[16] Bodin, P., Kreuter, M., Bake, B., and Fagevik Olse, M. (2003) Breathing patterns during breathing exercises in persons with tetraplegia. Spinal Cord, 41, 290-295.

[17] J. Derrickson, N. Ciesla, and N. Simpson. (1992) A comparison of two breathing exercise programs for patients with Quadriplegia. Physical Therapy, 72(11), 763-769. 